\title{
Does Diabetes Accelerate the Progression of Aortic Stenosis through Enhanced Inflammatory Response within Aortic valves?
}

\author{
Joanna Natorska, ${ }^{1,2,3}$ Ewa Wypasek, ${ }^{1,2}$ Grzegorz Grudzień, ${ }^{1,2}$ Dorota Sobczyk, ${ }^{2}$ \\ Grzegorz Marek, ${ }^{2}$ Grzegorz Filip, ${ }^{2}$ Jerzy Sadowski, ${ }^{1,2}$ and Anetta Undas ${ }^{1,2}$
}

\begin{abstract}
Diabetes predisposes to aortic stenosis (AS). We aimed to investigate if diabetes affects the expression of selected coagulation proteins and inflammatory markers in AS valves. Twenty patients with severe AS and concomitant type 2 diabetes mellitus (DM) and 40 well-matched patients without DM scheduled for valve replacement were recruited. Valvular tissue factor (TF), TF pathway inhibitor (TFPI), prothrombin, C-reactive protein (CRP) expression were evaluated by immunostaining and TF, prothrombin, and CRP transcripts were analyzed by real-time PCR. DM patients had elevated plasma CRP (9.2 [0.74-51.9] mg/l vs. 4.7 [0.59-23.14] mg/l, $p=0.009)$ and TF (293.06 [192.32-386.12] pg/ml vs. 140 [104.17-177.76] pg/ml, $p=0.003)$ compared to non-DM patients. In DM group, TF-, TFPI-, and prothrombin expression within valves was not related to demographics, body mass index, and concomitant diseases, whereas increased expression related to DM was found for CRP on both protein $(2.87[0.5-9] \%$ vs. $0.94[0-4] \%, p=0.01)$ and transcript levels $(1.3 \pm 0.61 v s .0 .22 \pm 0.43, p=0.009)$. CRP-positive areas were positively correlated with mRNA TF $(r=0.84, p=0.036)$. Diabetes mellitus is associated with enhanced inflammation within AS valves, measured by CRP expression, which may contribute to faster AS progression.
\end{abstract}

KEY WORDS: aortic stenosis; diabetes mellitus; C-reactive protein; inflammation.

\section{INTRODUCTION}

Aortic stenosis (AS) is the most frequent heart valve disease and the most common cause of heart valve replacement in the elderly [1]. It is characterized by massive fibrous thickening of valve leaflets and extensive focal calcification [2]. The development and progression of AS is an active inflammatory process, including disruption of the basement membrane, subendothelial accumulation of intracellular lipids, lipoproteins and molecular mediators of calcification, together with infiltration of the inflammatory cells and activation of local and systemic inflammation [3, 4]. The concept of AS as an atherosclerosis-like process

\footnotetext{
${ }^{1}$ Institute of Cardiology, Jagiellonian University Medical College, 80 Pradnicka Str., 31-202, Kracow, Poland

${ }^{2}$ John Paul II Hospital, Krakow, Poland

${ }^{3}$ To whom correspondence should be addressed at Institute of Cardiology, Jagiellonian University Medical College, 80 Pradnicka Str., 31-202, Kracow, Poland. E-mail: j.natorska@szpitaljp2.krakow.pl
}

is supported by a number of studies showing that development of AS is associated with cardiovascular risk factors such as smoking, hypercholesterolemia, and arterial hypertension [5, 6]. Diabetes mellitus (DM) has also been reported to represent a risk factor for AS however little is known about this association despite the strong impact of DM on coronary atherosclerosis [7]. Diabetes accompanied by inflammation is thought to play an important role in enhancing the pathophysiological mechanism of atherosclerosis [8]. It appears that CRP is directly involved in pro-atherogenic changes by amplification of vascular inflammatory response through complement activation, endothelial cell activation, and finally tissue damage [9]. Peripheral monocytes infiltrate early atherosclerotic lesions and show a high activation state in diabetes [10]. The proinflammatory state of diabetic monocytes is characterized by increased intranuclear factor kappa B $(\mathrm{NF} \kappa \mathrm{B})$, regulating pro-inflammatory genes [11] and induces an intravascular oxidative stress response [12], leading to 
TF expression [13]. TF was found to be an independent factor related to microvascular diabetic complications and it reflects endothelial dysfunction and procoagulant activity [14]. Recently, it has been shown that DM is associated with coronary artery calcium scores [15], aortic valve calcification [7] assessed by electronbeam computed tomography, and with faster AS progression [16]. It has been proposed that enhanced inflammation is implicated in progression of both DM [17] and AS [18]. Recent observations made in human aortic valves from patients with AS demonstrated increased expression of CRP and showed that the amount of pro-inflammatory proteins increased with the progression of AS [19]. Increased expression of tissue factor (TF), a major initiator of blood coagulation in vivo, associated with macrophage infiltration and neovascularization has been also demonstrated within the stenotic valves [20, 21].

To our knowledge, there have been no reports showing if DM affects the valvular expression of inflammatory and/or coagulation proteins in AS patients. Therefore, we sought to investigate whether DM influences the expression of CRP as well as TF, its inhibitor-TFPI (TF pathway inhibitor) and prothrombin in a representative patient population with advanced AS without clinically overt atherosclerotic vascular disease.

\section{MATERIALS AND METHODS}

\section{Patients}

A total of 60 consecutive patients ( 27 men and 33 women) undergoing isolated elective aortic valve replacement for severe AS (mean transvalvular gradient $\geq 40 \mathrm{mmHg}$ ) were recruited. The exclusion criteria were: acute infection, Valsalva sinus aneurysm or rheumatic AS, angiographically documented epicardial artery stenosis $>20 \%$ diameter, known cancer, autoimmune disorders, endocarditis, previous cardiac surgery, a history of myocardial infarction, stroke, venous thromboembolism, or bleeding. Patients who required additional surgical intervention or had other heart defects were ineligible.

Information on the presence or absence of cardiovascular risk factors, including arterial hypertension, hyperlipidemia, DM, and use of statins, angiotensinconverting enzyme inhibitors, $\beta$ blockers and acetylsalicylic acid was obtained before surgery. Patients were classified as having DM $(n=20)$ if they were receiving insulin $(n=7,35 \%)$, oral hypoglycemic agents $(n=13$, $65 \%$ ), and/or had fasting glucose levels $>7 \mathrm{mmol} / \mathrm{l}$. Only patients with DM without renal dysfunction (based on having at least two random fasting creatinine levels of $>110 \mu \mathrm{mol} / \mathrm{l}$ for male and $>80 \mu \mathrm{mol} / \mathrm{l}$ for female) were enrolled. Arterial hypertension was diagnosed based on a history of hypertension (blood pressure $>140$ / $90 \mathrm{mmHg}$ ) or preadmission antihypertensive treatment. Patients gave their informed consent and the study was approved by the University Bioethical Committee. Five aortic valves from age-matched apparently healthy subjects, obtained at autopsy, without morphological valvular or other cardiac disorders served as negative controls.

All measurements and tissue analysis were performed by technicians blinded to the origin of the samples. The samples were uncoded during statistical analysis.

\section{Echocardiography}

Transthoracic echocardiography was performed in each patient using a GE Vivid 7 ultrasound machine prior to surgery using conventional techniques in accordance with the European Society of Cardiology guidelines. The aortic valve area (AVA) was calculated using the standard continuity equation [22]. The transvalvular gradient was measured by Doppler echocardiography using the modified Bernoulli equation [22].

\section{Laboratory Tests}

Fasting venous blood was drawn from patients within $24 \mathrm{~h}$ before surgery between $5 \mathrm{AM}$ and $9 \mathrm{AM}$. Citrated blood samples $(9: 1$ of $0.129 \mathrm{M}$ citrate) were centrifuged at $3,000 \mathrm{rpm}$ at $20^{\circ} \mathrm{C}$ for $10 \mathrm{~min}$ and stored in aliquots at $-80^{\circ} \mathrm{C}$ until analysis. Glucose and creatinine were assayed by routine laboratory techniques. High-sensitivity C-reactive protein (CRP) was determined using immunoturbidimetry (Siemens Healthcare Diagnostics, Deerfield, IL, USA). Commercially available ELISA test was used to determine in citrated plasma TF (American Diagnostica Inc., Stamford, CT, USA) according to manufacturer's instructions. Intra-assay and inter-assay coefficients of variation were $<8 \%$.

\section{Analysis of Aortic Valves}

Diseased aortic valves were collected during surgery for valve replacement. Cryopreserved valves 
were cryosectioned on $10-\mu \mathrm{m}$ thick sections, as described before [20]. Slides were stored at $-20^{\circ} \mathrm{C}$ until immunostaining. Immunohistochemistry was performed on adjacent sections of aortic valves as described before [20]. Briefly, after endogenous peroxidase activity quenching and blocking of unspecific background, primary monoclonal antibodies were applied overnight at $4^{\circ} \mathrm{C}$ against human $\mathrm{TF}$ (R\&D Systems, Inc., Minneapolis, USA), TFPI (American Diagnostica Inc., Stamford, CT, USA), prothrombin (HyphenBioMed, Neuville-Sur-Oise, France) or CRP (Santa Cruz Biotechnology, Inc., CA, USA). Primary antibodies were followed by the corresponding secondary antibodies and by the avidin-biotin complex immunoperoxidase according to manufacturer's instructions (Santa Cruz Biotechnology Inc., CA, USA) and visualized using the diamninobenzidine. A negative control (without primary antibody incubation) was included routinely. Sections were viewed in light microscope (Zeiss, Berlin, Germany). Photomicrographs were taken using a Canon A640 camera and analyzed using the image analysis software cellAnalyst. The extent of immunoreactive areas of analyzed antigens was quantified in nine serial-step sections per valve as described by Lamprecht et al. [23].

\section{Nucleic Acid Extraction}

Human valves obtained during aortic valve replacement were immediately placed in RNALater (Qiagen, Chatsworth, CA, USA) and stored in liquid nitrogen. Frozen valve cups were pulverized using Mikro-Dismembrator S (Sartorius Stedim Biotech, Germany). Total RNA was isolated with the RNeasy Mini kit, according to the manufacturer's recommended protocols (Qiagen, Chatsworth, CA, USA) and digested with DNase (DNase I recombinant, RNase-Free, Qiagen, Chatsworth, CA, USA) following the manufacturer's instructions. The RNA concentration was estimated by A260 measurement, and the samples were stored at $-80^{\circ} \mathrm{C}$.

\section{Quantification of TF, Prothrombin, IL-6, and CRP Transcripts by Real-time PCR}

A total of $1 \mu \mathrm{g}$ of RNA from each individual valve cups was reverse-transcribed to single-strain cDNA using High Capacity RNA-to-cDNA Master Mix (Applied Biosystems) at $25^{\circ} \mathrm{C}$ for $5 \mathrm{~min}, 42^{\circ} \mathrm{C}$ for $30 \mathrm{~min}$ followed by $85^{\circ} \mathrm{C}$ for $5 \mathrm{~min}$. The cDNA was amplified with TaqMan Gene Expression Assays containing both primers and probe (TF, assay ID
Hs01076032_m1; prothrombin, Hs00354679_m1; IL-6, Hs00985639_m1; and CRP, Hs00265044_m1) on an ABI PRISM $^{\circledR} 7900$ HT Fast Real-Time PCR System (Applied Biosystems). The assays were from Applied Biosystems as inventoried or made-to-order.

Beta-actin (Hs99999903_m1, human ACTB Endogenous Control FAM/MGB Probe, Non-Primer Limited; Applied Biosystems) was used as a housekeeping gene. To analyze the obtained data, the comparative threshold cycle (CT) method was applied [24]. Briefly, the amount of a target gen, normalized to $\mathrm{ACTB}$, and relative to a calibrator (normal valve leaflet), is given by $2^{-\Delta \Delta C T}$, where $\Delta \Delta C_{T}=\Delta C_{T}$ of a sample (the $\mathrm{C}_{\mathrm{T}}$ of the target gene of AS valves subtracted from the $\mathrm{C}_{\mathrm{T}}$ of $\left.\mathrm{ACTB}\right)-\Delta \mathrm{C}_{\mathrm{T}}$ of calibrator.

\section{Statistical Analysis}

Values are expressed as mean (SD) or median (interquartile range) or otherwise stated. The KolmogorovSmirnov test was used to assess conformity with a normal distribution. Pairwise comparisons were made using the Tukey's test for continuous variables and the $\chi^{2}$ test for proportions. The Mann-Whitney $U$ test was used to compare non-normally distributed variables between two groups. The Spearman's correlation coefficient was calculated to evaluate associations between variables. A value of $p<0.05$ was considered statistically significant.

\section{RESULTS}

Baseline characteristics of patients from DM and non-DM groups are shown in Table 1.

Patients with DM had elevated plasma CRP (9.2 [0.74-51.9] mg/l vs. $4.7[0.59-23.14] \mathrm{mg} / \mathrm{l}, p=0.009)$ and TF (293.06 [192.32-386.12] pg/ml vs. 140 [104.17-177.76] $\mathrm{pg} / \mathrm{ml}, p=0.003$ ) levels compared to non-DM patients.

\section{Immunohistochemistry and Immunofluorescence}

Focal areas of subendothelial thickening were seen in the AS valves from both DM and non-DM groups. Immunohistochemical staining of aortic valve tissue showed the abundance of TF antigen at the aortic side of the leaflets, colocalizing with TFPI-positive areas in both DM and non-DM groups; however, there were no statistically significant differences in TF or TFPI expression within valves from DM $v s$. non-DM group $(20.43 \pm 10.44 \%$ vs. $17.46 \pm 9.44 \%, p=0.56$; and $46.17 \pm 12.61 \%$ vs. $39.53 \pm 10.4 \%, p=0.63$, respectively). 
Table 1. Baseline Characteristics of Patients with (DM) and without (non-DM) Diabetes Mellitus

\begin{tabular}{llll}
\hline & DM $(n=20)$ & non-DM $(n=40)$ & $P$ value \\
\hline Male, $n(\%)$ & $11(55)$ & $16(40)$ & 0.2 \\
Age, years & $66.42 \pm 7.7$ & $62.23 \pm 11.06$ & 0.44 \\
Body mass index $\left(\mathrm{kg} / \mathrm{m}^{2}\right)$ & $31.08 \pm 5.3$ & $29.6 \pm 5.51$ & \\
Echocardiography & & & \\
Mean aortic gradient $(\mathrm{mmHg})$ & $61.87 \pm 25.26$ & $55.12 \pm 22.29$ & 0.18 \\
Maximum aortic gradient (mmHg) & $86.9 \pm 24.6$ & $93.01 \pm 24.7$ & 0.11 \\
LVEF $(\%)$ & $53.13 \pm 16.39$ & $57.44 \pm 12.58$ & 0.33 \\
AVA $\left(\mathrm{cm}^{2}\right)$ & $0.73 \pm 0.19$ & $0.72 \pm 0.23$ & \\
Treatment & & & 0.93 \\
Beta blockers, $n(\%)$ & $11(55)$ & $20(50)$ & 0.61 \\
Acetylsalicylic acid, $n(\%)$ & $13(65)$ & $23(57)$ & 0.77 \\
Statins, $n(\%)$ & $10(50)$ & $16(40)$ & 0.28 \\
ACEIs, $n(\%)$ & $6(30)$ & $21(52)$ & 0.01 \\
\hline
\end{tabular}

Data are given as mean $\pm \mathrm{SD}$, median (IQR) or number (percentage)

$L V E F$ left ventricular ejection fraction, $A V A$ aortic valve area, $A C E I s$ angiotensin-converting enzyme inhibitors

Prothrombin antigen was detected in both groups and the expression of this antigen did not differ between DM and non-DM valves $(26.05 \pm 12.47 \%$ vs. $23.42 \pm$ $13 \%, p=0.72)$. Of note, prothrombin was colocalized with TF and TFPI; however, areas positive for prothrombin were also visible in other TF-free regions. CRP expression within stenotic valves was detected in fibrosa but not in valvular spongiosa (Fig. 1). Compared to non-DM, valves from DM group were characterized by increased expression of CRP $(0.94[0-4] \%$ vs. $2.87[0.5-9] \%, p=0.01)$, (Fig. 2a).

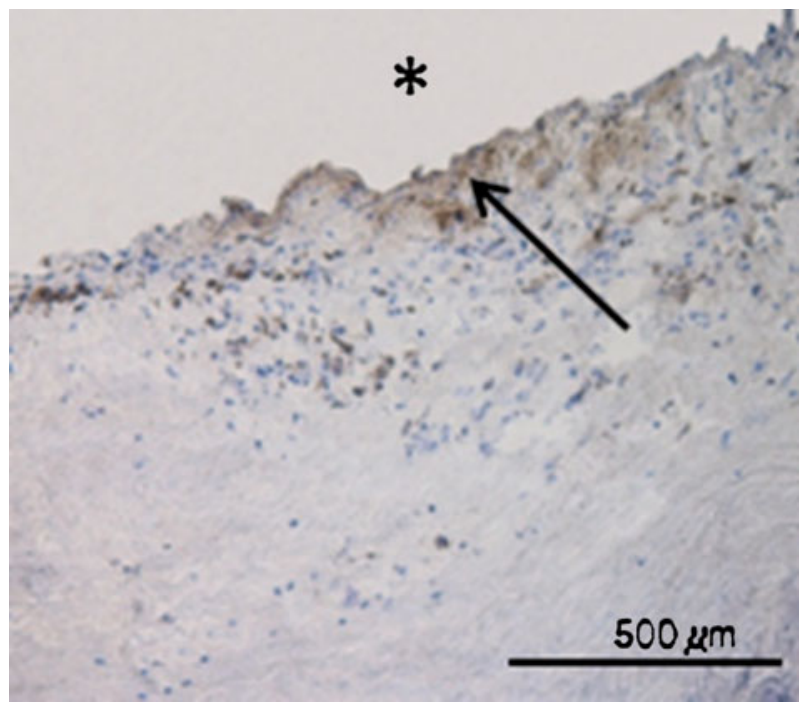

Fig. 1. Photomicrograph of representative valve lesion showing immunodetection of C-reactive protein within stenotic aortic valves in patients with diabetes mellitus. Immunopositive areas are stained brown. Asterisk shows aortic side of the leaflet.
There was no age-, gender-, or smoking-related differences in the percentage of areas positive for TF, TFPI, prothrombin-, or CPR antigens. There was a positive correlation between TF- and TFPI-positive areas in both $\mathrm{DM}$ and non-DM groups $(r=0.77, p=0.002 ; r=$ $0.74, p<0.0001$, respectively) and between $\mathrm{TF}$ and prothrombin $(r=0.76, p=0.004 ; r=0.51, p=0.003$, respectively). Interestingly, CRP expression within valves was positively correlated with TF expression in DM but not in non-DM group $(r=0.83, p=0.0001 ; r=$ $0.19, p=0.063$, respectively). In both DM and non-DM groups, there were positive correlations of TF with maximal $(r=0.7, p=0.005 ; r=0.5, p=0.008$, respectively) and mean ( $r=0.58, p=0.03 ; r=0.71, p=0.004$, respectively) gradients and inverse correlation of AVA ( $r=-0.67, p=0.004 ; r=-0.93, p=0.001$, respectively), but not with LVEF $(r=0.2, p=0.094 ; r=0.14, p=0.45)$. Positive correlations of prothrombin with maximal $(r=0.54, p=0.02 ; r=0.85, p=0.004)$ and mean $(r=$ $0.62, p=0.03 ; r=0.87, p=0.003)$ gradients and inverse correlation with AVA ( $r=-0.42, p=0.04 ; r=-0.88$, $p=0.004$ ) were also found. No significant correlations were found between analyzed protein expressions and plasma levels of CRP and TF.

\section{Valvular mRNA Expression}

Analysis of relative gene expression data using real-time quantitative PCR has shown increased expression of CRP ( $1.3 \pm 0.61$ vs. $0.22 \pm 0.43, p=0.009)$ but not TF $(2.6 \pm 0.83 v s .1 .7 \pm 0.16, p=0.39)$ or prothrombin $(0.31 \pm 1.33$ vs. $0.27 \pm 0.04, p=0.8)$ in DM group compared to non-DM group (Fig. 2b). Moreover, positive 

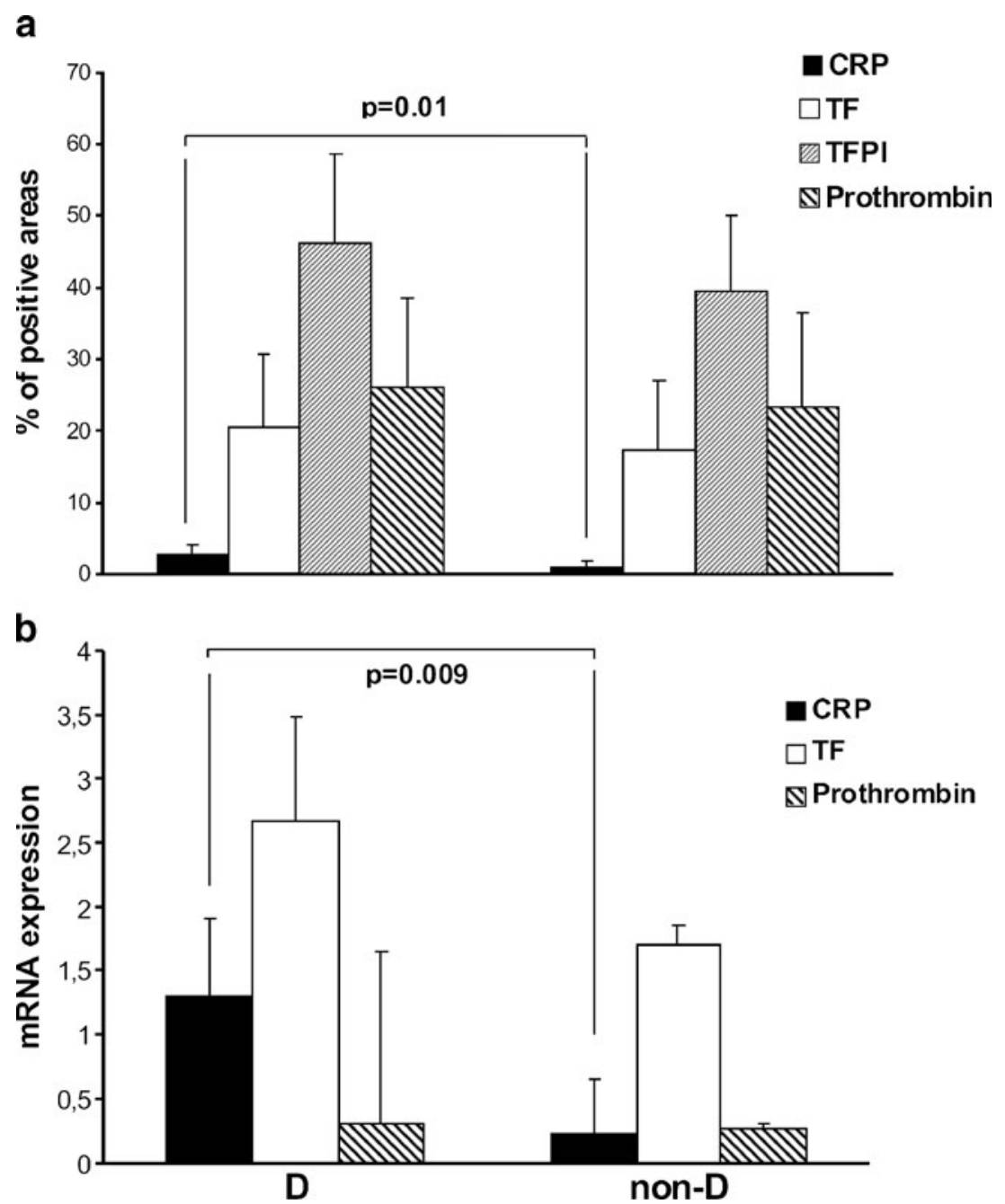

Fig. 2. Quantitative analysis of a immunostained C-reactive protein $(C R P)$, tissue factor $(T F)$, tissue factor pathway inhibitor (TFPI) and prothrombin, and $\mathbf{b}$ mRNA levels of CRP, TF, and prothrombin, within aortic valves in patients with aortic stenosis and with (DM) or without (non-DM) diabetes mellitus. Bars represent means \pm SD or median (IQR). A $p$ value $<0.05$ was considered statistically different.

correlations of mRNA for TF with areas immunopositive for CRP were found in DM patients $(r=0.84, p=0.036)$.

No significant correlations were found between analyzed mRNA expressions and plasma levels of CRP and TF.

\section{DISCUSSION}

To our knowledge, the current study is the first to show that in subjects with AS, DM augments the expression of crucial pro-inflammatory protein-CRP within aortic valves. Our major findings can be summarized as follows: (1) DM is associated with an increased percentage of CRP-positive areas within stenotic valves, which is correlated with areas positive for TF, and (2) mRNA for TF is positively correlated with the percentage of CRP-immunopositive areas in patients with DM.

Disputing the classic dogma that CRP is synthesized exclusively by the liver in response to inflammatory cytokines, recent reports have shown that CRP is produced in various non-hepatic tissue and cells. Of major relevance, CRP expression has been detected in the arterial tissue and increased levels of CRP mRNA have been documented in atherosclerotic plaque [25]. Consisting with these findings, stimulatory effect of oxidized low-density lipoproteins on CRP synthesis 
by freshly isolated human monocytes has been also shown [26].

It has been proposed that inflammation plays a crucial intermediatory role in the pathogenesis of glucose disorders in adults [27, 28], thereby linking diabetes with a number of commonly coexisting conditions thought to originate through inflammatory mechanisms, such as CRP. In this study, elevated plasma CRP level was associated with the development of diabetes, supporting the previous data [17, 29] and expanding our previous data, showing stenosed valves inflammation manifested by huge macrophage infiltrations [20] and emphasizing the role of local inflammation within stenosed valves. An independent association of CRP with AS progression has been also previously documented by Galante et al. [30] arising a new understanding of the physiological role of CRP in stenotic valves, through promoting local proinflammatory effects. The reported immunoregulatory functions of CRP include enhancement of macrophages and $\mathrm{T}$ lymphocytes activity with subsequent tissue damage, complement fixation, and modulation of platelet activation [31, 32], which leads to valve cusp degradation and AS progression. Our previous study has shown that macrophage infiltrations is colocalized with large amounts of TF within stenosed valves [20], which suggests that macrophages play a vital role in $\mathrm{TF}$ synthesis and are important in a cross-talk between inflammatory and coagulatory processes in this disease. In other studies, we have shown that within the stenotic valves, the TF molecules are active and lead to thrombin formation [33]. Giving the data that thrombin has been shown to enhance valve calcification and AS progression [21], it might be suggested that faster AS progression in DM patients is an effect of enhancing inflammatory and coagulation processes within valve cusps, which leads to increased valve leaflets calcification.

\section{Study Limitations}

First, the number of DM patients enrolled in this study was limited. However, both groups were well matched. Additionally, the severity, and the duration of DM were not the same in patients from DM group. Second, the present study is focused on the valves obtained from patients with severe AS. Once the valve is heavily calcified, inflammation may play less of a role in progression of AS than in a valve with less severe disease and the influence of DM on AS may be masked to some extent. Therefore, our results cannot be evaluated using the current approach and extrapolated on subjects with mild AS. Third, patients from DM group had BMI $>30 \mathrm{~kg} / \mathrm{m}^{2}$, and obesitymediated CRP production is an important mechanism for systemic elevation of this protein [29], which could partially influence the results. Finally, glycated hemoglobin, a retrospective DM indicator, has not been measured which could be the reason of the lower detection of DM [34].

In conclusion, diabetes was found to be associated with increased inflammation within AS valves, as measured by CRP expression. Further studies are warranted to determine whether screening for aortic valve disease should be recommended in patients with diabetes mellitus. Also, further investigations are needed to define whether interventions that directly target insulin resistance in diabetes might retard or reverse the progression of AS.

\section{ACKNOWLEDGMENTS}

This study has been supported by a grant of Polish Ministry of Science (\# N N402 383338, to A.U.).

Conflict of interest. None.

Open Access. This article is distributed under the terms of the Creative Commons Attribution Noncommercial License which permits any noncommercial use, distribution, and reproduction in any medium, provided the original author(s) and source are credited.

\section{REFERENCES}

1. Otto, C.M., B.K. Lind, D.W. Kitzman, B.J. Gersh, and D.S. Sisovick. 1999. Associations of aortic valve sclerosis with cardiovascular mortality and morbidity in the elderly. The New England Journal of Medicine 341: 142-147.

2. Ortlepp, J.R., M. Pillich, F. Schmitz, V. Mevissen, R. Koos, S. Weiss, L. Stork, R. Dronskowski, G. Langebartels, R. Autschbach, V. Brandenburg, S. Woodruff, J.J. Kaden, and R. Hoffmann. 2006. Lower serum calcium levels are associated with greater calcium hydroxyapatite deposition in native aortic valves of male patients with severe calcific aortic stenosis. The Journal of Heart Valve Disease 15: 502-508.

3. Yetkin, E., and J. Waltenberger. 2009. Molecular and cellular mechanisms of aortic stenosis. International Journal of Cardiology 135: 4-13. 
4. Mohty, D., P. Pibarot, J.P. Després, C. Côté, B. Arsenault, A. Cartier, P. Cosnay, C. Couture, and P. Mathieu. 2008. Association between plasma LDL particle size, valvular accumulation of oxidized LDL, and inflammation in patients with aortic stenosis. Arteriosclerosis, Thrombosis, and Vascular Biology 28: 187-193.

5. Pate, G.E. 2002. Association between aortic stenosis and hypertension. The Journal of Heart Valve Disease 11: 612-614.

6. Ngo, M.V., J.S. Gottdiener, R.D. Fletcher, D.J. Fernicola, and B.J. Gersh. 2001. Smoking and obesity are associated with progression of aortic stenosis. The American Journal of Geriatric Cardiology 10: 86-90.

7. Katz, R., N.D. Wong, R. Kronmal, J. Takasu, D.M. Shavelle, J.L. Probstfield, A.G. Bertoni, M.J. Budoff, and K.D. O'Brien. 2006. Features of the metabolic syndrome and diabetes mellitus as predictors of aortic valve calcification in the Multi-Ethnic Study of Atherosclerosis. Circulation 113: 2113-2119.

8. Jawień, J. 2008. New insights into immunological aspects of atherosclerosis. Polskie Archiwum Medycyny Wewnettrznej 118: $127-131$.

9. Miłosz, D., L. Czupryniak, M. Saryusz-Wolska, G. Zasadzińska, A. Borkowska, E. Cieplucha, K. Chizyński, and J. Loba. 2007. Adiponectinemia, inflammatory process activity, and endothelial dysfunction in patients with type 2 diabetes and acute coronary syndrome with ST elevation in relation to the severity of lesions in the coronary arteries. Polskie Archiwum Medycyny Wewnetrznej 117: 343-349.

10. Cipolletta, C., K.E. Ryan, E.V. Hanna, and E.R. Trimble. 2005. Activation of peripheral blood CD14+ monocytes occurs in diabetes. Diabetes 54: 2779-2786.

11. Voleti, B., and A. Agrawal. 2005. Regulation of basal and induced expression of $\mathrm{C}$-reactive protein through an overlapping element for OCT-1 and NF-kappaB on the proximal promoter. Journal of Immunology 175: 3386-3390.

12. Guerrero-Romero, F., and M. Rodríguez-Morán. 2006. Hypomagnesemia, oxidative stress, inflammation, and metabolic syndrome. Diabetes/Metabolism Research and Reviews 22: 471-476.

13. Bierhaus, A., T. Illmer, M. Kasper, T. Luther, P. Quehenberger, H. Tritschler, P. Wahl, R. Ziegler, M. Müller, and P.P. Nawroth. 1997. Advanced glycation end products-mediated induction of tissue factor in cultured endothelial cells is dependent on RAGE. Circulation 96: 2262-2271.

14. Sommeijer, D.W., H.R. Hansen, R. van Oerle, K. Hamulyak, A.P. van Zanten, E. Meesters, H.M. Spronk, and H. Cate. 2006. Soluble tissue factor is a candidate marker for progression of microvascular disease in patients with Type 2 diabetes. Journal of Thrombosis and Haemostasis 4: 574-580.

15. Wolfe, M.L., N. Iqbal, W. Gefter, E.R. Mohler 3rd, D.J. Rader, and M.P. Reilly. 2002. Coronary artery calcification at electron beam computed tomography is increased in asymptomatic type 2 diabetics independent of traditional risk factors. Journal of Cardiovascular Risk 9: 369-376.

16. Kamalesh, M., C. Ng, H. El Masry, G. Eckert, and S. Sawada. 2009. Does diabetes accelerate progression of calcific aortic stenosis? European Journal of Echocardiography 10: 723-725.

17. Pradhan, A.D., J.E. Manson, N. Rifai, J.E. Buring, and P.M. Ridker. 2001. C-reactive protein, interleukin 6, and risk of developing type 2 diabetes mellitus. JAMA 286: 327-334.

18. Sanchez, P.L., and A.M. Mazzone. 2006. C-reactive protein in aortic valve disease. Cardiovascular Ultrasound 4: 37-40.

19. Toli, K., K.I. Paraskevas, M.V. Poulakou, G. Agrogiannis, N. Kavantzas, V. Xanthopoulos, D.G. Iliopoulos, I. Mantas, A. Papachristodoulou, E. Patsouris, D.P. Mikhailidis, and D.N. Perrea. 2008. Association between plasma levels and immunolocalization of cytokines in heart valve lesions: a possible target for treatment? Expert Opinion on Therapeutic Targets 12: 1209-1215.

20. Natorska, J., G. Marek, M. Hlawaty, D. Sobczyk, J. Sadowski, W. Tracz, and A. Undas. 2009. Evidence for tissue factor expression in aortic valves in patients with aortic stenosis. Polskie Archiwum Medycyny Wewnettrznej 119: 636-642.

21. Breyne, J., F. Juthier, D. Croseaux, S. Marechaux, Ch Zawadzki, E. Jeanpierre, A. Ung, P.V. Ennezat, S. Susen, E. Van Belle, H. Le Marec, A. Vincentelli, T. Le Tourneau, and B. Jude. 2010. Atherosclerotic-like process in aortic stenosis: Activation of the tissue factor-thrombin pathway and potential role through osteopontin alternation. Atherosclerosis 213: 369-376.

22. Teirstein, P., M. Yeager, P.G. Yock, and R.L. Popp. 1986. Doppler echocardiographic measurement of aortic valve area in aortic stenosis: a noninvasive application of the Gorlin formula. Journal of the American College of Cardiology 8: 1059-1065.

23. Lamprecht, M.R., D.M. Sabatini, and A.E. Carpenter. 2007. Cell profiler: Versalite software for automated biological image analysis. BioTechniques 42: 71-75.

24. Clark-Greuel, J.N., J.M. Connolly, E. Sorichillo, N.R. Narula, H.E. Rapoport, E.R. Mohler, J.H. Gorman, R.C. Gorman, and R.J. Levy. 2007. Transforming growth factor beta-1 mechanisms in aortic valve calcification: Increased alkaline phosphatase and related events. The Annals of Thoracic Surgery 7: 946-953.

25. Yasojima, K., C. Schwab, E.G. McGeer, and P.L. McGeer. 2001. Generation of C-reactive protein and complement components in atherosclerotic plaques. American Journal of Pathology 158: 1039-1051.

26. Mugabo, Y., L. Li, and G. Renier. 2010. The connection between C-reactive protein (CRP) and diabetic vasculopathy. Focus on preclinical findings. Current Diabetes Reviews 6: 27-34.

27. Barzilay, J.I., L. Abraham, S.R. Heckbert, M. Cushman, L.H. Kuller, H.E. Resnick, and R.P. Tracy. 2011. The relation of markers of inflammation to the development of glucose disorders in the elderly: The Cardiovascular Health Study. Diabetes 50: 2384-2389.

28. Sjöholm, A., and T. Nyström. 2006. Inflammation and the etiology of type 2 diabetes. Diabetes/Metabolism Research and Reviews 22: 4-10.

29. Sweat, V., V. Starr, H. Bruehl, A. Arentoft, A. Tirsi, E. Javier, and A. Convit. 2008. C-reactive protein is linked to lower cognitive performance in overweight and obese women. Inflammation 31: 198-207.

30. Galante, A., A. Pietroiusti, M. Vellini, P. Piccolo, G. Possati, M. De Bonis, R.L. Grillo, C. Fontana, and C. Favalli. 2001. C-reactive protein is increased in patients with degenerative aortic valvular stenosis. Journal of the American College of Cardiology 38: 1078-1082.

31. Kaden, J.J., C.E. Dempfle, R. Grobholz, C.S. Fischer, D.C. Vocke, R. Kiliç, A. Sarikoç, R. Piñol, S. Hagl, S. Lang, M. Brueckmann, and M. Borggrefe. 2005. Inflammatory regulation of extracellular matrix remodeling in calcific aortic valve stenosis. Cardiovascular Pathology 14: 80-87.

32. Marnell, L., C. Mold, and T.W. Du Clos. 2005. C-reactive protein: ligands, receptors and role in inflammation. Clinical Immunology 117: 104-111.

33. Natorska, J., G. Marek, M. Hlawaty, J. Sadowski, W. Tracz, and A. Undas. 2011. Fibrin presence within aortic valves in patients with aortic stenosis: Association with in vivo thrombin generation and fibrin clot properties. Thrombosis and Haemostasis 105: 254-260.

34. Drzewoski, J., and A. Drozdowska. 2010. Could glycated hemoglobin be used as a diagnostic tool in diabetes mellitus? Polskie Archiwum Medycyny Wewnętrznej 20: 109-114. 